\title{
Evaluación de activación conductual en universitarios que consumen mariguana: estudio piloto
}

\author{
Emmanuel Said Baeza-Torres', Sara Eugenia Cruz Morales², Jennifer Lira Mandujano² \\ I Programa de Maestría y Doctorado, Universidad Nacional Autónoma de México, Ciudad de México, México \\ 2 Facultad de Estudios Superiores Iztacala, Universidad Nacional Autónoma de México, Edo. México, México
}

\section{RESUMEN}

Introducción: en México el consumo de mariguana ha aumentado en estudiantes universitarios, lo cual ocasiona problemas en atención, concentración, ansiedad, depresión, dependencia y problemas cardiorrespiratorios. Las intervenciones psicológicas como la intervención breve motivacional (IBM) y la terapia cognitivo conductual (TCC) han reportado algunas dificultades, como un tamaño del efecto entre bajo y medio, disminución de consumo sin llegar a abstinencia y la heterogeneidad en el uso de técnicas tanto cognitivas como conductuales. El uso de técnicas conductuales, principalmente en activación conductual, ha reportado efectividad en el tratamiento de ansiedad, depresión y más recientemente de alcohol y tabaco. Objetivo: evaluar el tamaño del efecto de un tratamiento en Activación Conductual (AC-M) en la abstinencia del consumo de mariguana en unidades, ocasiones y cantidad. Método: en una muestra $(n=8)$ de estudiantes universitarios se aplicó el tratamiento en AC-M que incluye técnicas de análisis funcional, planificación y establecimiento de metas, manejo de contingencias, entrenamiento en habilidades y prevención de recaídas. Se realizó un diseño de caso único multielemento con ocho réplicas y se calculó el tamaño del efecto a través del índice de no solapamiento de todos los pares. Resultados: se obtuvo un tamaño del efecto alto en el patrón de consumo, oscilando entre $91.10 \%$ y $100 \%$, donde cinco universitarios lograron abstinencia.

Discusión y conclusiones: el tratamiento en AC-M obtuvo un tamaño de efecto alto en la abstinencia y disminución del consumo de mariguana resultados similares a lo reportado en alcohol y tabaco.

Palabras clave: activación conductual, consumo de mariguana, universitarios.

\begin{abstract}
Introduction: In Mexico, marijuana consumption has increased in adults and university students, triggering problems in attention, concentration, anxiety, depression, dependence, and cardio-respiratory difficulties. Psychological interventions like brief motivational treatment (BMT) and cognitive behavioral therapy (CBT) have reported some difficulties such as a low and medium effect size, decreased consumption without reaching abstinence, and heterogeneity in the use of both cognitive and behavioral techniques. The use of behavioral techniques in Behavioral Activation has reported effectiveness in the treatment of anxiety, depression, and recently in alcohol and tobacco. Objective: to evaluate the effect size of a Behavioral Activation treatment in abstinence of marijuana (BA-M) measured in units, occasions, and quantity of consumption. Method: in a sample of university students $(n=8)$, the intervention of behavioral activation was applied, which includes functional analysis, planning and goal setting, contingency management, skills training, and relapse prevention. It was carried out a multielement single case design with eight replications, calculating the effect size through the non-overlapping index of all pairs. Results: high effect size was obtained in the consumption pattern, ranging between $91.10 \%$ and $100 \%, 5$ university students achieved abstinence. Discussion and conclusions: treatment in BA-M obtained a high effect size in abstinence and a decrease in marijuana abuse these results are similar to those reported in alcohol and tobacco.
\end{abstract}

Keywords: behavioral activation, marijuana abuse, university students.

\footnotetext{
Autor de correspondencia:

Emmanuel Said Baeza Torres

Programa de Maestría y Doctorado en Psicología, Universidad Nacional Autónoma de México. FES-Iztacala, UNAM. Av. Barrios, No. 1, Los Reyes Iztacala Tlalnepantla, Edo. México, 54090, México. Teléfono: 55 3706-4915

Correo electrónico: said.bato@yahoo.es

Recibido: 14 de enero de 2021

Aceptado: 12 de mayo de 2021

doi: 10.28931/riiad.2021.2.02
} 


\section{INTRODUCCIÓN}

El consumo de mariguana ha incrementado a nivel internacional de acuerdo con la Oficina de Naciones Unidas contra la Droga y el Delito (UNODC, por sus siglas en inglés, United Nations Office on Drugs and Crime, 2017), se estima que del 2012 al 2015, cerca de 183 millones de personas (25\% hombres y $19 \%$ mujeres) consumieron al menos una vez en el último año, 57.2\% reportó el consumo de otras drogas ilegales ([UNODC], 2017).

En México, la Encuesta Nacional de Consumo de Drogas, Alcohol y Tabaco 2016, en adultos de 18 a 36 años, reportó que el consumo de mariguana "alguna vez" y en el "último año" incrementó en hombres (de $9.3 \%$ pasó a $20 \%$ ), (de $2.2 \%$ aumentó a $5.9 \%$ ) y mujeres (de $1.1 \%$ pasó a $6.1 \%$ ), (de $0.2 \%$ incrementó a $1.3 \%$ ) respectivamente (Villatoro-Velázquez et al., 2015). En países de Latinoamérica como Colombia, Brasil, Perú, Bolivia, Venezuela, según la Comisión Interamericana para el Control del Abuso de Drogas en su informe del Uso de Drogas en las Américas ([ClCAD], 2015), se observó una tendencia similar en la población universitaria.

En el informe de los efectos sociales y consecuencias para la salud del consumo de mariguana, la Organización Panamericana de la Salud (OPS), reporta problemas en atención, concentración, accidentes automovilísticos, violencia, relaciones sexuales sin condón, trastornos de ansiedad, depresión, dependencia, intoxicación, problemas cardiorrespiratorios, esquizofrenia y suicidio (OPS, 2018). En universitarios se reportan problemas legales en el campus universitario, deserción y ausentismo escolar, materias reprobadas y consumo de otras drogas ilegales como cocaína (Feingold et al., 2015; Skidmore et al., 2016).

En cuanto al abordaje del trastorno por uso de mariguana (TUM) se cuenta con tratamientos psicológicos y farmacológicos (Gates et al., 2016). En la farmacoterapia no existe un fármaco aprobado por la Administración de Alimentos y Medicamentos (FDA) para el tratamiento del TUM (Sherman \& McRae-Clark, 2016); sin embargo, la $\mathrm{N}$-acetilcisteína y la gabapentina han sido útiles para el abordaje de la intoxicación por uso de mariguana (Cooper, 2016; Hermanns-Clausen et al., 2013).

Respecto a los tratamientos psicológicos, al ser la terapia cognitivo-conductual (TCC) el tratamiento más empleado para consumo de mariguana con un tamaño del efecto entre bajo y medio; se desconoce cuáles de sus técnicas se relacionan con la abstinencia al consumo (Gates et al., 2016). Por un lado, las técnicas cognitivas y entrevista motivacional (EM) se relacionan con la reduc- ción del consumo sin llegar a la abstinencia (McCambridge \& Strang, 2004). Además del desconocimiento de los mecanismos de cambio por los cuales la EM subyace el cambio de consumo (Apodaca \& Longabaugh 2009), las técnicas conductuales como el entrenamiento en habilidades (EH) para rechazo al consumo ante emociones displacenteras se han relacionado con la disminución de una posible recaída (Gulloa et al., 2017). El manejo de contingencias (MC) ha demostrado resultados prometedores, por lo que se recomienda evaluarlo dentro de un tratamiento conductual enfocado en la abstinencia (Litt et al., 2013).

La Activación Conductual (AC), tratamiento conductual desarrollado inicialmente para depresión (Ferster, 1973), ha demostrado su eficacia para depresión y ansiedad en comparación con la terapia cognitiva (Ekers et al., 2008; 2011). Postula bajo un modelo operante que la depresión es consecuente de un incremento en conductas reforzadas negativamente, y ante un aumento de actividades opuestas a la depresión reforzadas positivamente aumenta la probabilidad de ponerse en contacto con reforzadores naturales (Ferster, 1973). La AC consta de técnicas como análisis funcional, planeación de actividades, establecimiento de metas, monitoreo de actividades (Martell et al., 2013). Kanter et al. (2010), mencionan que el MC y el EH son técnicas que se pueden añadir según la problemática a tratar.

La AC fue evaluada inicialmente en la adherencia al tratamiento residencial en poliusuarios, donde se encontraron resultados en el mantenimiento de la abstinencia y mayores tasas de adherencia (Daughters et al., 2017). En estudios piloto se ha mostrado evidencia para consumo de alcohol y tabaco, comórbido con ansiedad y depresión (Mac-Pherson et al., 2017; Reynolds et al., 2011), ya que, de manera similar a la depresión, el consumo de sustancias se puede explicar tanto por reforzamiento positivo (emociones agradables, consumo experimental) como negativo (ansiedad y depresión; Kedzior \& Laeber, 2014; Szerman et al., 2013). Por ello se recomienda continuar las investigaciones en estudios piloto su factibilidad con otras sustancias como la mariguana (Bowen et al., 2009; Vujanovic et al., 2017).

Por tal motivo, el objetivo del presente estudio es evaluar, mediante un diseño de caso único multielemento con ocho réplicas, el tamaño del efecto del tratamiento de activación conductual para mariguana (AC-M) sobre la disminución del patrón de consumo de mariguana en unidades, ocasiones y cantidad a lo largo del tratamiento y seguimientos un mes, tres y seis meses después. 


\section{MÉTODO}

\section{Participantes}

Muestra no probabilística $(n=8)$ de hombres entre 18 y 24 años de edad con consumo actual de mariguana y que estuvieran cursando estudios universitarios. Se presentaron voluntariamente a diversos centros de atención psicológica de consulta externa de la UNAM, ya que deseaban dejar de fumar mariguana. Se les proporcionó un consentimiento informado en el que aceptaron las políticas de privacidad, como la confidencialidad de la información, los resultados del estudio con fines de investigación, la duración del tratamiento y se especificó que podían declinar en cualquier momento.

\section{Criterios de inclusión y exclusión}

Criterios de inclusión: universitarios de cualquier licenciatura entre 18 y 24 años de edad, con consumo de mariguana en los últimos tres meses, consecuencias asociadas al consumo leve, moderado y/o severo, y estar de acuerdo con el consentimiento informado. Criterios de exclusión: consumo de otras drogas legales e ilegales que requirieran intervención breve o intensiva, niveles moderado y/o severo de consecuencias asociadas a otras sustancias, estar paralelamente en otra intervención psiquiátrica o psicológica y haber presentado algún intento suicida en los últimos tres meses.

\section{Instrumentos}

Entrevista conductual: se obtienen datos sociodemográficos, historia y patrón de consumo, y principales consecuencias asociadas (Baeza, et al., en preparación).

Línea base retrospectiva (LIBARE): su objetivo es obtener un registro retrospectivo de consumo a un mes antes de iniciar el tratamiento (Sobell \& Sobell 1992, adaptado por Echeverría \& Ayala, 1997).

Cronograma de actividades: su objetivo es obtener un registro de la frecuencia de actividades opuestas al consumo y patrón de consumo (Barraca \& Pérez, 2015).

Cuestionario de cuantificación del consumo de mariguana CCC-M: estima el gramaje a través del método de consumo, circunferencia, longitud, tope y volumen (Ortega, 2016).

Cuestionario de abuso de drogas (CAD-20): medir la percepción que el individuo tiene hacia las consecuencias de su consumo de drogas (Villalobos et al., 2015).

Prueba de detección de consumo de alcohol, tabaco y sustancias (ASSIST v3.1): el objetivo es ayudar a la identificación temprana de riesgos para la salud, problemas asociados al uso de sustancias y nivel de la intervención requerida. Elaborada por la Organización Mundial de la Salud [2010] y adaptada a México (Tiburcio et al., 2016). DrugCheck Dip Drug Test: inmunoensayo para la detección cualitativa del metabolito $\Delta$ 9-tetrahidrocannabinol a una concentración de $50 \mathrm{ng} / \mathrm{ml}$ en orina.

\section{Diseño}

Diseño de caso único multielemento con ocho réplicas; se utilizó ya que es necesario corroborar, antes de evaluar su eficacia y efectividad, la factibilidad del tratamiento en AC-M en el abordaje del consumo de mariguana como nueva variable dependiente (Bowen et al., 2009). Además, permite observar en cada sesión del tratamiento el cambio del patrón de consumo del universitario visto como su propio control experimental y con ello asegurar que dicho cambio sea producto del tratamiento. En caso contrario, el curso del tratamiento se puede modificar para lograr la abstinencia de manera satisfactoria (Perone \& Hursh, 2013; Sanz \& García-Vera, 2015).

\section{Procedimiento}

A través del muestreo propositivo/intencional (Kerlinger \& Howard, 2002), mediante difusión se invitó a recibir el tratamiento a estudiantes provenientes de la Facultad de Estudios Superiores Iztacala, UNAM, del centro de servicios psicológicos "Dr. Guillermo Dávila" y del centro de prevención en adicciones "Dr. Héctor Ayala Velázquez", UNAM. Los estudiantes que aceptaron participar fueron contactados para iniciar la sesión de admisión y evaluación en la que se realizó una entrevista y evaluación para determinar si cubrían los criterios de inclusión y exclusión evaluados con el CAD-20 y ASSIST v3.1. La estructura y técnicas del tratamiento de AC-M (ver Tabla 1) se describen a partir de tres fases: 1. Admisión y evaluación (una sesión), 2. Tratamiento (cuatro sesiones), y 3. Seguimientos (un mes, tres y seis meses) después de haber terminado la intervención. Los seguimientos tienen el objetivo de trabajar futuras situaciones de riesgo que pudieran afectar el mantenimiento de la abstinencia y además corroborar la misma con la prueba de orina DrugCheck® Dip Drug, cuyo rango de detección es de 10 a 30 días en consumidores crónicos.

\begin{abstract}
Análisis
El tamaño del efecto de la aplicación del tratamiento se determinó a partir de las unidades, ocasiones y cantidad de consumo, con el índice de No solapamiento de todos los pares (NAP) desarrollado por Parker y Vannest (2009), en el que se compara por pares todos los solapamientos posibles de la línea base con el tratamiento y seguimientos con la fórmula: NAP $=($ Numero de pares
\end{abstract}


Tabla 1

Estructura de la intervención en Activación conductual para consumidores de mariguana (AC-M)

\begin{tabular}{|c|c|c|c|}
\hline Sesión & Objetivo & Técnicas aplicadas & Instrumentos y materiales \\
\hline $\begin{array}{l}\text { Admisión y } \\
\text { evaluación }\end{array}$ & $\begin{array}{l}\text { Explicar el modelo contextual del trata- } \\
\text { miento, evaluar el patrón de consumo } \\
\text { de mariguana y niveles de ansiedad y } \\
\text { depresión. }\end{array}$ & $\begin{array}{l}\text { Entrevista } \\
\text { Evaluación } \\
\text { Retroalimentación } \\
\text { Monitoreo de actividades }\end{array}$ & $\begin{array}{l}\text { Entrevista conductual } \\
\text { Consentimiento informado } \\
\text { Inventario ansiedad, depresión rasgo-estado } \\
\text { y Folleto 1. AC-M } \\
\text { CAD-20, ASSIST, CCC-M }\end{array}$ \\
\hline Sesión 1 & $\begin{array}{l}\text { Identificar y establecer actividades } \\
\text { opuestas al consumo en función de } \\
\text { metas establecidas en cada una de las } \\
\text { cinco áreas de vida. }\end{array}$ & $\begin{array}{l}\text { Análisis funcional } \\
\text { Planeación de actividades } \\
\text { Establecimiento de metas }\end{array}$ & $\begin{array}{l}\text { Cronograma de actividades } \\
\text { CCC-M y planes de acción } \\
\text { Folleto } 2 \text {. Áreas de vida y planificación de } \\
\text { actividades }\end{array}$ \\
\hline Sesión 2 & $\begin{array}{l}\text { Establecer y autoadministrar recom- } \\
\text { pensas agradables con la finalidad de } \\
\text { facilitar la realización de actividades } \\
\text { opuestas al consumo en las que el } \\
\text { estudiante tenga dificultad en realizar. }\end{array}$ & $\begin{array}{l}\text { Manejo de contingencias } \\
\text { Planeación de actividades }\end{array}$ & $\begin{array}{l}\text { Cronograma de actividades } \\
\text { CCC-M y planes de acción } \\
\text { Folleto } 3 \text {. Recompensando el cumplimiento } \\
\text { de mis actividades }\end{array}$ \\
\hline Sesión 3 & $\begin{array}{l}\text { Entrenar en comunicación asertiva } \\
\text { para el rechazo al consumo y proponer } \\
\text { la realización de otras actividades. }\end{array}$ & $\begin{array}{l}\text { Entrenamiento en habili- } \\
\text { dades de comunicación } \\
\text { Planeación de actividades }\end{array}$ & $\begin{array}{l}\text { Cronograma de actividades } \\
\text { CCC-M y planes de acción } \\
\text { Folleto 4. Comunicación asertiva }\end{array}$ \\
\hline Sesión 4 & $\begin{array}{l}\text { Generalizar las técnicas aprendidas a } \\
\text { otros contextos y preparar al estudiante } \\
\text { para futuras situaciones de riesgo. Eva- } \\
\text { luar patrón de consumo y sintomatolo- } \\
\text { gía ansiosa y depresiva. }\end{array}$ & $\begin{array}{l}\text { Prevención de recaídas } \\
\text { Evaluación } \\
\text { Retroalimentación }\end{array}$ & $\begin{array}{l}\text { CCC-M } \\
\text { Planes de acción } \\
\text { Inventario ansiedad y depresión rasgo-estado } \\
\text { Folleto 5. Prevención de recaídas }\end{array}$ \\
\hline Seguimientos & $\begin{array}{l}\text { Identificar situaciones de riesgo que } \\
\text { pudieran provocar alguna recaída y } \\
\text { evaluar patrón de consumo y niveles } \\
\text { de ansiedad y depresión. }\end{array}$ & $\begin{array}{l}\text { Entrevista } \\
\text { Evaluación } \\
\text { Retroalimentación }\end{array}$ & $\begin{array}{l}\text { Entrevista de seguimiento } \\
\text { Inventario ansiedad y depresión rasgo-estado } \\
\text { LIBARE }\end{array}$ \\
\hline
\end{tabular}

sin solapamiento $)+(0.5 \times$ Número de empates $)$ / Total de número de pares de datos comparado con la línea base y el tratamiento $\times 100$.

\section{RESULTADOS}

En las características de la muestra se encontró una edad promedio de 22.6 años de edad; edad de inicio de consumo $X=17.7$; edad problema $X=20.12$; intentos de dejar el consumo $X=2.75$. Respecto al nivel de problemas asociados al consumo de mariguana de acuerdo con el CAD20 un participante reportó nivel bajo, cuatro reportaron nivel moderado, dos con nivel sustancial y uno con nivel severo. De acuerdo con el ASSIST sobre el nivel de intervención, tres participantes reportaron intervención breve y cinco requirieron intervención intensiva (ver Tabla 2).
Respecto a las unidades (Figura 1), ocasiones (Figura 2) y cantidad de consumo (Figura 3 ), se puede observar una línea base estable (A), excepto por el sujeto 7. En la fase de tratamiento (B) se observa una disminución de cada una de las variables para todos los sujetos. Sin embargo, al concluir el tratamiento mediante la prueba de orina DrugCheck@ Dip Drug se encontró que cuatro de ellos (2, 4, 7 y 8 ) reportaron resultados positivos. En la fase de seguimientos $(\mathrm{C})$ en comparación con (B), se observa una disminución de las tres variables en todos los sujetos. No obstante, tres de ellos (4, 7 y 8) aún presentaron consumo en el último seguimiento corroborado con la prueba de orina.

Con respecto al tamaño del efecto (ver Tabla 3) se muestra un efecto grande para cada una de las tres variables en los ocho sujetos. 
Tabla 2

Características de los sujetos participantes

\begin{tabular}{|c|c|c|c|c|c|c|c|}
\hline Participante & Edad & Edad inicio & Edad problema & Sexo & CAD-20 & ASSIST & $\begin{array}{c}\text { Intentos anteriores para } \\
\text { dejar de fumar }\end{array}$ \\
\hline 1 & 19 & 15 & 17 & $M$ & 12 & 28 & 3 \\
\hline 2 & 24 & 16 & 24 & $M$ & 10 & 31 & 3 \\
\hline 3 & 24 & 23 & 24 & $M$ & 3 & 15 & 0 \\
\hline 4 & 22 & 17 & 21 & M & 6 & 24 & 2 \\
\hline 5 & 24 & 15 & 17 & M & 10 & 30 & 3 \\
\hline 6 & 23 & 26 & 20 & $M$ & 6 & 26 & 5 \\
\hline 7 & 21 & 15 & 20 & M & 13 & 27 & 2 \\
\hline 8 & 24 & 15 & 18 & $M$ & 17 & 34 & 4 \\
\hline
\end{tabular}

Figura 1

Unidades de consumo semanal de los sujetos participantes a lo largo del tratamiento y seguimientos

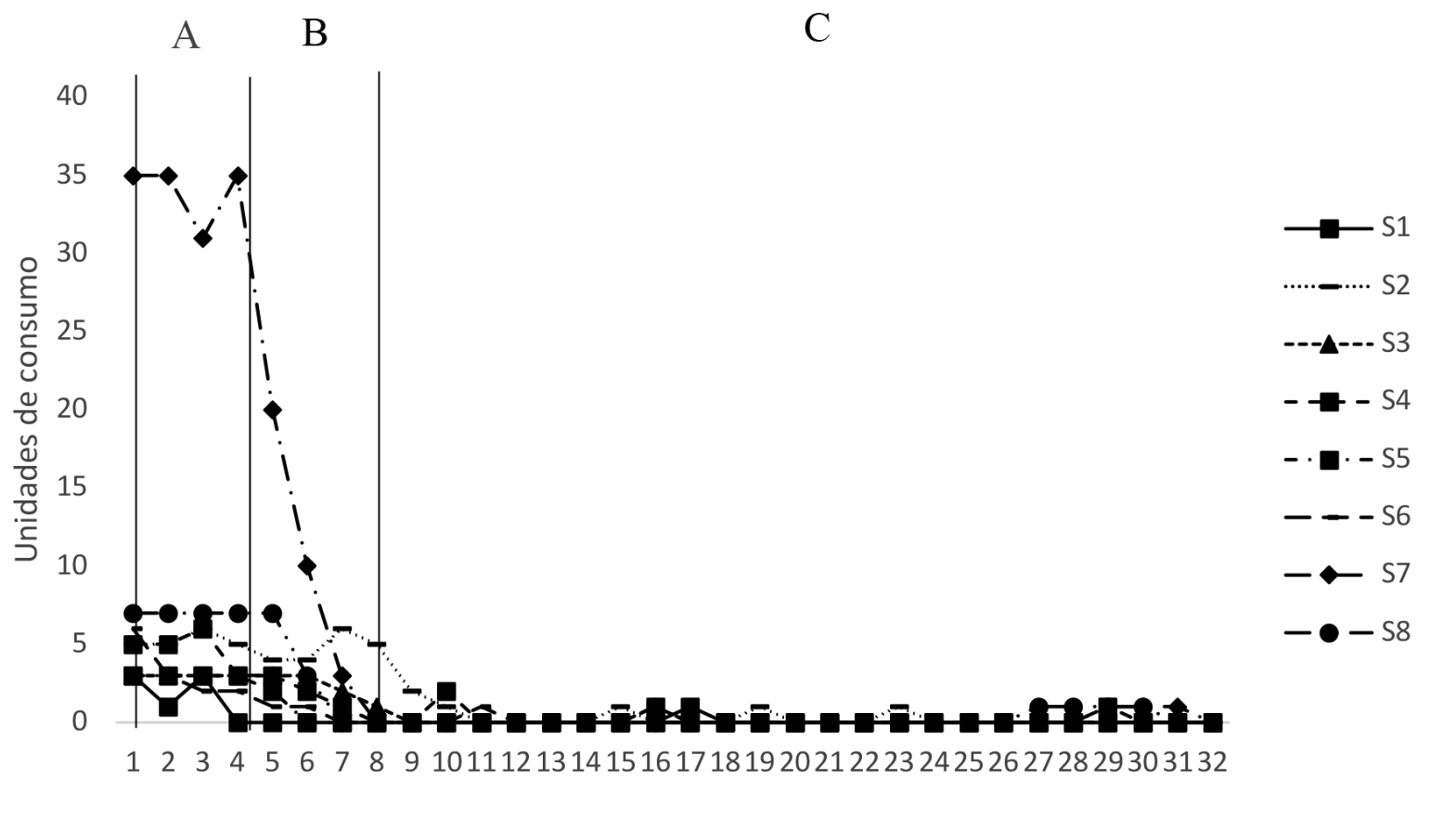


Figura 2

Ocasiones de consumo semanal de los sujetos participantes a lo largo del tratamiento y seguimientos

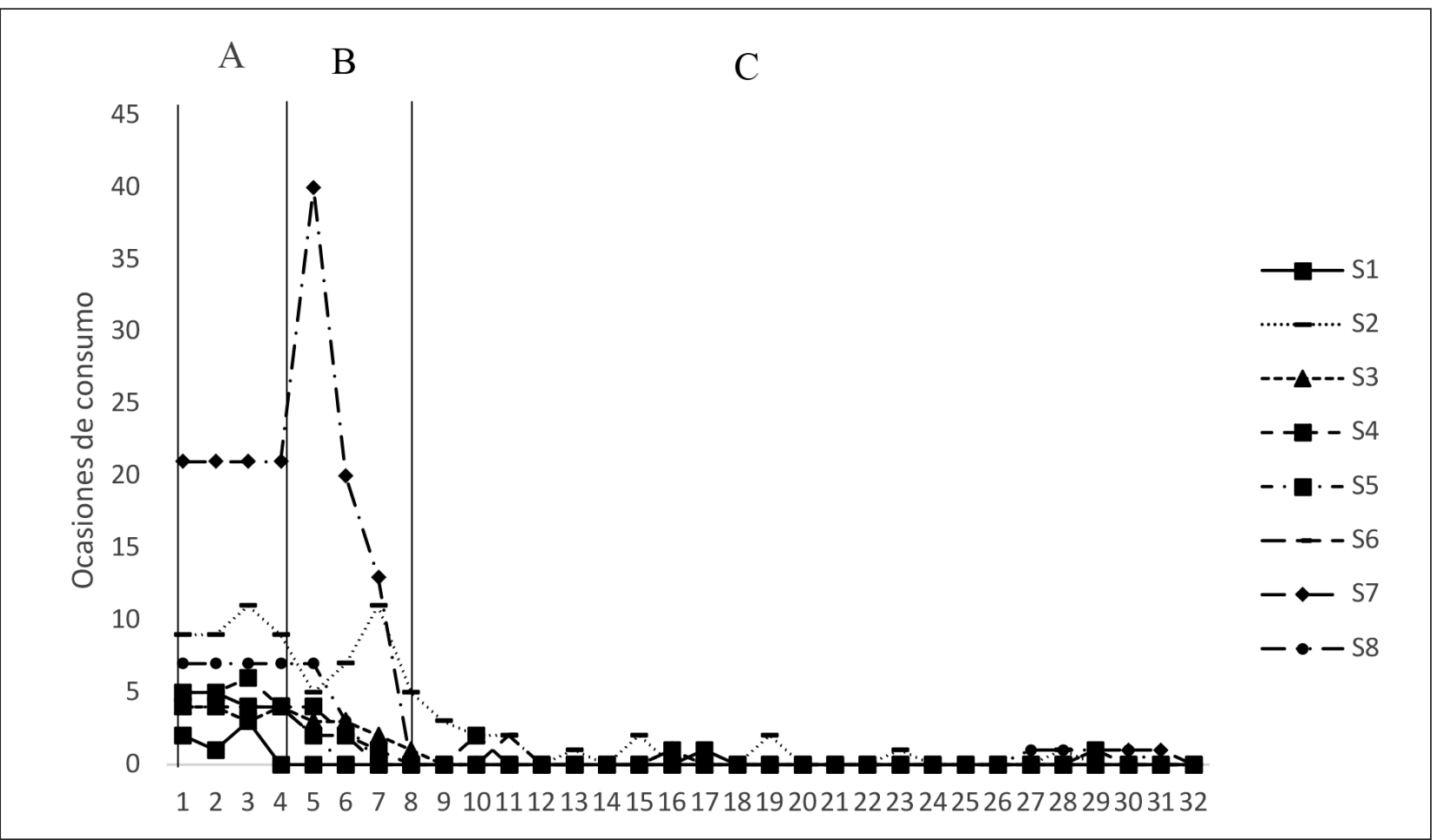

Figura 3

Cantidad de consumo semanal de los sujetos participantes a lo largo del tratamiento y seguimientos

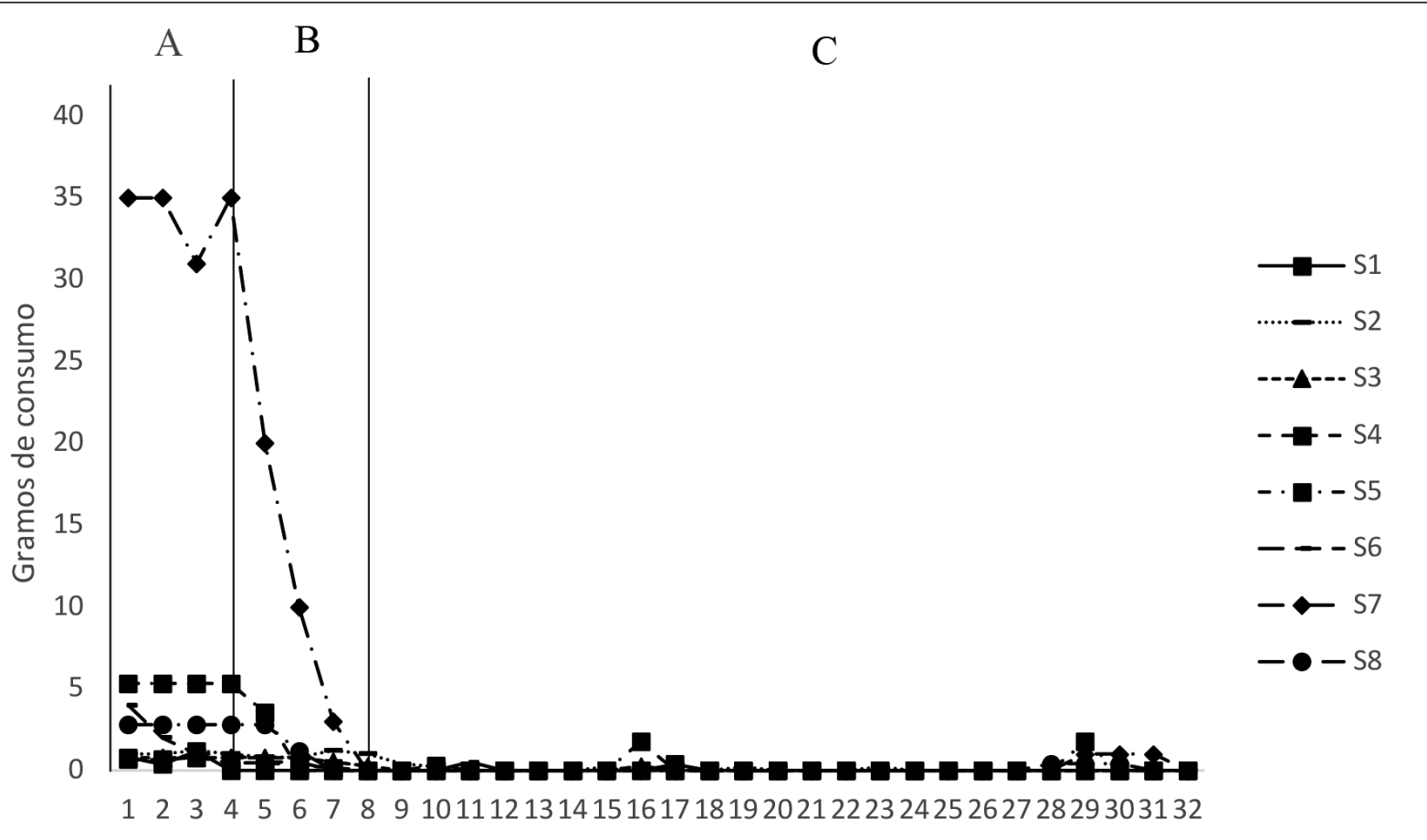


Tabla 3

Tamaño del efecto de los sujetos participantes

\begin{tabular}{|c|c|c|}
\hline$N$ & Valores & Interpretación \\
\hline \multicolumn{3}{|l|}{ Sujeto 1} \\
\hline Unidades & $100 \%$ & \\
\hline Ocasiones de consumo & $100 \%$ & Efecto grande \\
\hline Gramos & $99.5 \%$ & \\
\hline \multicolumn{3}{|l|}{ Sujeto 2} \\
\hline Unidades & $99.5 \%$ & \\
\hline Ocasiones de consumo & $99.10 \%$ & Efecto grande \\
\hline Gramos & $99.5 \%$ & \\
\hline \multicolumn{3}{|l|}{ Sujeto 3} \\
\hline Unidades & $99.10 \%$ & \\
\hline Ocasiones de consumo & $99.10 \%$ & Efecto grande \\
\hline Gramos & $99.10 \%$ & \\
\hline \multicolumn{3}{|l|}{ Sujeto 4} \\
\hline Unidades & $99.5 \%$ & \\
\hline Ocasiones de consumo & $100 \%$ & Efecto grande \\
\hline Gramos & $99.5 \%$ & \\
\hline \multicolumn{3}{|l|}{ Sujeto 5} \\
\hline Unidades & $100 \%$ & \\
\hline Ocasiones de consumo & $100 \%$ & Efecto grande \\
\hline Gramos & $100 \%$ & \\
\hline \multicolumn{3}{|l|}{ Sujeto 6} \\
\hline Unidades & $100 \%$ & \\
\hline Ocasiones de consumo & $100 \%$ & Efecto grande \\
\hline Gramos & $100 \%$ & \\
\hline \multicolumn{3}{|l|}{ Sujeto 7} \\
\hline Unidades & $100 \%$ & \\
\hline Ocasiones de consumo & $99.10 \%$ & Efecto grande \\
\hline Gramos & $100 \%$ & \\
\hline \multicolumn{3}{|l|}{ Sujeto 8} \\
\hline Unidades & $99.5 \%$ & \\
\hline Ocasiones de consumo & $100 \%$ & Efecto grande \\
\hline Gramos & $99.5 \%$ & \\
\hline
\end{tabular}

\section{DISCUSIÓN}

Con base en el objetivo planteado para unidades, ocasiones y cantidad de consumo, se obtuvo un rango entre $91.10 \%$ y $100 \%$, equivalente a un tamaño del efecto grande en AC-M según Parker y Vannest (2009). Esto se refleja en la abstinencia conseguida en cinco estudiantes, identificada con el cronograma de actividades y verificada con la prueba de orina DrugCheck $\Theta$ Dip Drug. A pesar de que tres de ellos (4, 7 y 8 ) mantuvieron un consumo bajo sin llegar a la abstinencia, éste fue menor en comparación con la línea base. Dichos resultados concuerdan con la literatura en estudios piloto para alcohol y tabaco (McPherson et al., 2017; Reynolds et al., 2011). Además, en comparación con la línea base, mantener un consumo menor en el último seguimiento puede prevenir el riesgo de una recaída, es decir volver al patrón de consumo original (Useche et al., 2017).

Estos resultados aportan una primera aproximación en la evidencia científica en función a un tratamiento conductual sobre la abstinencia del consumo de mariguana, ya que de acuerdo con la literatura la TCC con componentes cognitivos, conductuales y motivacionales es el tratamiento más empleado para el abordaje de dicha problemática con un tamaño del efecto entre bajo y medio (Gates et al., 2016). En México, la intervención breve para usuarios de mariguana (IBUM) es el tratamiento más empleado (Palafox et al., 2017), en el que se observan resultados similares en la reducción del consumo sin llegar a la abstinencia $(F=54.37, p<0.000)$ en una muestra heterogénea de estudiantes universitarios y de educación media superior (Echeverría et al., 2013).

Respecto al tipo de intervención y consecuencias asociadas, de acuerdo con los criterios de derivación a tratamientos intensivos en la IBUM, el participante debe presentar un nivel de problema sustancial o severo (CAD-20) y un nivel de tratamiento intensivo (ASSIST v3.1; Medina et al., 2012; OMS, 2010). De los participantes que obtuvieron un nivel de problemática sustancial (1 y 7), severo (8) y tratamiento intensivo (1, 2, 5, 7 y 8), la mayoría logró la abstinencia, excepto los participantes 7 y 8 , quienes sólo disminuyeron su consumo en comparación con línea base.

Estos resultados pueden ser explicados de acuerdo con las bases teóricas del tratamiento en activación conductual (AC-M), donde Barraca y Pérez (2015) mencionan que la conducta que es reforzada negativamente como la depresión, ansiedad y consumo de mariguana; o bien, reforzada positivamente, como suele ser el caso en el consumo experimental de mariguana y de las emociones agradables (Preedy, 2017), se suprimirá si se ejecutan e incrementan actividades opuestas al consumo, además 
la conducta eliminada se mantendrá a través del tiempo mediante el uso de MC enfocada en la abstinencia (Kanter et al., 2010; Litt et al., 2013).

Respecto al tipo de análisis utilizado, los diseños de caso único generalmente se han evaluado a través del análisis visual de los datos o pruebas no paramétricas de aleatorización (Sanz \& García-Vera, 2015). Una de sus limitaciones es llegar a cometer el error tipo I, ya que se ha encontrado que en el $25 \%$ de las veces se considera efectivo un tratamiento que no lo es (Campbell \& Herzinger, 2010). Por otro lado, las pruebas no paramétricas requieren decidir al azar el momento en que se aplica el tratamiento y por razones clínicas, prácticas y éticas esto no suele ser posible en la práctica clínica (Bono \& Arnau, 2014). Por tal motivo, una de las aportaciones para superar esta brecha es el índice para la estimación del tamaño del efecto basado en el no solapamiento de los datos entre las fases (Bono \& Arnau, 2014), que permite la construcción de intervalos de confianza y poner a prueba el tamaño del efecto frente a una hipótesis de nulidad, lo cual es útil con los diseños de caso único con pocos sujetos (Parker et al., 2011).

\section{CONCLUSIÓN}

El tratamiento en AC-M tuvo un tamaño del efecto mayor tanto en unidades, ocasiones y cantidad de consumo, relacionado con las tasas de abstinencia en cinco de los ocho participantes. De acuerdo con la literatura, el abordar esta problemática bajo componentes conductuales aporta evidencia científica de su eficacia al lograr la abstinencia en consumidores de mariguana sin la implementación de componentes cognitivos o motivacionales (Gates et al., 2016).

Aunque hubo cinco casos que requerían intervención intensiva según los criterios de derivación del programa de IBUM y el ASSIST v3.1 (Medina et al., 2012; OMS, 2010), tres de ellos lograron la abstinencia, esto podría argumentar y apoyar el uso de una intervención breve en AC-M para casos que pudieran requerir un tratamiento con un mayor número de sesiones. Sin embargo, se requiere realizar más investigación para delimitar qué casos pudieran ser candidatos a este tipo de intervención y evitar generalizar resultados.

El abordaje de esta problemática bajo el condicionamiento operante, podría tener un impacto en otras problemáticas (ansiedad y depresión) asociadas al consumo de mariguana, ya que parten del supuesto teórico de ser conductas que son reforzadas negativamente (Vujanovic et al., 2017). Es importante añadir que el consumo de mariguana puede ser reforzado tanto negativamente como positivamente y esto da pauta a que la aplicación e incremento de actividades opuestas al consumo sean actividades reforzadas positivamente.
La OPS (2018) ha sugerido hacer investigación con indicadores de cantidad de consumo de mariguana. Una de las aportaciones del presente estudio es el uso del instrumento de cuantificación de consumo de mariguana, el cual proporciona información sobre la cantidad en gramos de uso en los participantes (Ortega, 2016), además de una primera aproximación al abordaje del consumo bajo un tratamiento conductual que ha reportado evidencia en otras problemáticas (Bowen et al., 2009), y el tener una muestra homogénea en rango de edad de estudiantes universitarios sólo con consumo de mariguana, ya que la ausencia de dichas variables ha sido una de las dificultades encontradas en la evaluación de tratamientos para consumo de sustancias (Rojas et al., 2011).

A pesar de haber encontrado un tamaño del efecto alto, una de las limitaciones del diseño de caso único es no poder generalizar los resultados o concluir que el estudiante se ha recuperado de su problemática a tratar (Sanz \& García-Vera, 2015). Para futuras investigaciones se recomienda evaluar la intervención en muestras más grandes; realizar estudios de eficacia comparando el tratamiento de AC-M con la IBUM; incluir mediciones de ansiedad y depresión a lo largo del tratamiento y seguimientos, y realizar evaluación entre componentes para poder identificar qué técnicas tuvieron impacto en la abstinencia, o si es necesario añadir otras técnicas conductuales para manejo de ansiedad, considerando dicha variable, además de la depresión, como precipitadores del consumo.

\section{FUENTES DE FINANCIAMIENTO}

Emmanuel Said Baeza Torres recibió beca con el número 697082, por parte del Consejo Nacional de Ciencia y Tecnología (CONACYT) como alumno en el Programa de Maestría y Doctorado en Psicología, Doctorado en Psicología Clínica y de la Salud.

\section{DECLARACIONES DE LOS AUTORES}

Los autores declaran no tener ningún tipo de conflicto de interés.

\section{REFERENCIAS}

Apodaca, T. R., \& Longabaugh, R. (2009). Mechanisms of change in motivational interviewing: A review and preliminary evaluation of the evidence. Addiction, 104(5), 705-715. doi: 10.1111/j.13600443.2009.02527.x

Barraca, J., \& Pérez, M. (2015). Activación conductual para el tratamiento de la depresión. Editorial Síntesis. 
Baeza, E. S., Cruz-Morales, S. E., \& Mandujano, J. (2019). Eficacia de activación conductual para dejar de consumir mariguana en universitarios (Tesis Doctoral no publicada).

Bono, R., \& Arnau, J. (2014). Diseños de caso único en ciencias sociales y de la salud. Editorial Síntesis.

Bowen, D. J., Kreuter, M., Spring, B., Cofta-Woerpel, L., Linnan, L., Weiner, D., Bakken, S., Kaplan, C. P., Squiers, L., Fabrizio, C., \& Fernandez, M. (2009). How we design feasibility studies. American Journal of Preventive Medicine, 36(5), 452-457. doi: 10.1016/j.amepre.2009.02.002

Campbell, J. M., \& Herzinger, C. V. (2010). Statistics and single subject research methodology. En: D. L. Gast, (Eds.), Single Subject Research Methodology in Behavioral Sciences (pp. 417-453). Routledge.

Comisión Interamericana para el Control del Abuso de Drogas. (2015). Informe sobre uso de drogas en las Américas. Observatorio Interamericano de Drogas: Washington, D.C. Recuperado de www.cicad.oas.org/cicaddocs/document.aspx?ld=3209

Cooper, Z. D. (2016). Adverse Effects of Synthetic Cannabinoids: Management of Acute Toxicity and Withdrawal. Current Psychiatry Reports, 18(5), 52. doi: 10.1007/s11920-016-0694-1.

Daughters, S. B., Magidson, J. F., Anand, D., Seitz-Brown, C. J., Chen Y., \& Baker, S. (2017). The effect of a behavioral activation treatment for substance use on post-treatment abstinence: a randomized controlled trial. Addiction, 113, 535-544. doi: 10.1111/add.14049

Echeverría, L., \& Ayala, H. E. (1997). Auto registro del consumo de alcohol y otras drogas: traducción y adaptación. Universidad Nacional Autónoma de México.

Echeverría, L., Carrascoza C., \& Medina, M. A. (2013). Intervención breve para consumidores de mariguana: Factores asociados con la deserción. En: Aspectos culturales, sociales y preventivos de las adicciones. Conaculta.

Ekers, D., Richards, D., \& Gilbody, S. (2008). A meta-analysis of randomized trials of behavioural treatment of depression. Psychological Medicine, 38(5), 611-623. doi: 10.1017/S0033291707001614

Ekers, D., Godfrey, C., Gilbody, S., Parrott, S., Richards, D. A., Hammond, D., \& Hayes, A. (2011). Cost utility of behavioural activation delivered by the non-specialist. The British Journal of Psychiatry, 199(6), 510-511. doi: 10.1192/bjp.bp.110.090266.

Feingold, D., Weiser, M., Rehm, J., \& Lev-Ran, S. (2015). The association between cannabis use and mood disorders: A longitudinal study. Journal of Affective Disorders, (1)172, 211-218. doi: 10.1016/j.jad.2014.10.006

Ferster, C. B. (1973). A functional analysis of depression. American Psychologist, 28(10), 857-870. doi: 10.1037/h0035605

Gates, P. J., Sabioni, P., Copeland, J., Le-Foll, B., \& Gowing, L. (2016). Psychosocial interventions for cannabis use disorder. The Cochrane Database of Systematic Reviews, 1(5), 2-147. doi: 10.1002/14651858.CD005336.pub4

Gulloa, M. J., Matveeva, M., Feeneya, G. F., Young, R. M., \& Connora, J. P. (2017). Social cognitive predictors of treatment outcome in cannabis dependence. Drug and Alcohol Dependence, 1(170), 74-81. doi: 10.1016/j.drugalcdep.2016.10.030.
Hermanns-Clausen, M., Kneisel, S., Szabo, B., \& Auwärter, V. (2013). Acute toxicity due to the confirmed consumption of synthetic cannabinoids: clinical and laboratory findings. Addiction, 108(3), 534-544. doi: 10.1111/j.1360-0443.2012.04078.x.

Kanter, J. W., Manos, R. C., Bowe, W. M., Baruch, D. E., Busch, A. M., \& Rusch, L. C. (2010). What is behavioral activation? A review of the empirical literature. Clinical Psychology Review, 30(6), 608-20. doi: 10.1016/j.cpr.2010.04.001

Kedzior, K. K., \& Laeber, L. T. (2014). A positive association between anxiety disorders and cannabis use or cannabis use disorders in the general population--a meta-analysis of 31 studies. BMC Psychiatry, 14(1), doi: 10.1186/1471-244X-14-136

Kerlinger, F., \& Howard, B. (2002). Investigación del comportamiento (4a. ed.). Mc Graw-Hill.

Litt, M. D., Kadden, R. M., \& Petry, N. M. (2013). Behavioral Treatment for Marijuana Dependence: Randomized Trial of Contingency Management and Self-Efficacy Enhancement. Addictive Behaviors, 38(3), 1764-1775. doi:10.1016/j.addbeh.2012.08.011.

Mac-Pherson, L., Collado, A., Ninnemann, A., \& Hoffman, E. (2017). Development of a Behavioral Activation-Based Intervention for $\mathrm{Ci}$ garette-Smoking Young Adults. Cognitive and Behavioral Practice, 24, 101-114. doi: 10.1016/j.cbpra.2016.03.004

Martell, C. R., Dimidjian, S., \& Herman-Dunn, R. (2013). Activación Conductual para la Depresión: Una guía clínica. Editorial Desclée de Brouwer.

McCambridge, J., \& Strang, J. (2004). The efficacy of single-session motivational interviewing in reducing drug consumption and perceptions of drug-related risk and harm among young people: results from a multi-site cluster randomized trial. Addiction, 99(1), 39-52. doi: 10.1111/j.1360-0443.2004.00564.x

Medina, M. A., Chávez, M. E., López, F., Félix, V., Gordillo, T., \& Morales, S. (2012). Manual del Terapeuta para la Aplicación de la Intervención Breve para Usuarios de Marihuana. Secretaría de Salud: Centro Nacional para la Prevención y el Control de las Adicciones.

Organización Mundial de la Salud. (2010). The Alcohol, Smoking and Substance Involvement Screening Test (ASSIST): manual for use in primary care. Recuperado de https://apps.who.int/iris/ handle/10665/44320

Organización Panamericana de la Salud. (2018). Efectos sociales y para la salud del consumo de cannabis sin fines médicos. Recuperado de https://iris. paho.org/bitstream/handle/10665.2/34944/9789275319925_spa.pdf?sequence=1\&isAllowed =y

Ortega, B. E. (2016). Relación entre la conducta autocontrolada y la conducta adictiva en procedimientos de "abstención" y demora de la gratificación (tesis de maestría). Universidad Nacional Autónoma de México.

Palafox, G., Morales, S., Félix, V., López, F., Martínez, K., \& Vázquez, J. L. (2017). Transferencia de las intervenciones breves para la prevención de recaídas en adicciones. Recuperado de http:// www.cicad.oas.org/cicaddocs/Document.aspx?ld=4757 
Parker, R. I., \& Vannest, K. (2009). An improved effect size for single case research: Non Overlap of All Pairs. Behavior Therapy, 40(4), 357-367. doi: 10.1016/j.beth.2008.10.006

Parker, R. I., Vannest, K. J., \& Davis, J. L. (2011). Effect size in single-case research: a review of nine nonoverlap techniques. Behavior Modification, 35(4), 303-322. doi: 10.1177/0145445511399147

Perone, M., \& Hursh, D. E. (2013). Single-case experimental designs. En G. J. Madden, W. V. Dube, T. D. Hackenberg, G. P. Hanley, \& K. A. Lattal (Eds.), APA handbook of behavior analysis, Vol. 1. Methods and principles (pp. 107-126). American Psychological Association. doi: 10.1037/13937-005

Preedy, A. (2017). Handbook of Cannabis and Related Pathologies: Biology, Pharmacology, Diagnosis, and Treatment. Academic Press.

Reynolds, E. K., MacPherson, L., Baruch, D. E., Tull, M. T., \& Lejuez, C. W. (2011) Integration of the Brief Behavioral Activation Treatment for Depression (BATD) Into a College Orientation Program: Depression and Alcohol Outcomes. Journal of Counseling Psychology, 58(4), 555-564. doi: 10.1037/a0024634

Rojas, E., Real, T., García-Silberman, S., \& Medina-Mora, M. E. (2011). Revisión sistemática sobre tratamiento de adicciones en México. Salud Mental, 34(4), 351-365.

Sanz, J., \& García-Vera, M. P. (2015). Técnicas para el análisis de diseños de caso único en la práctica clínica: Ejemplos de aplicación en el tratamiento de víctimas de atentados terroristas. Clínica y Salud, 26(3), 167-180. doi: 10.1016/j.clysa.2015.09.004

Sherman, B. J., \& McRae-Clark, A. L. (2016). Treatment of Cannabis Use Disorder: Current Science and Future Outlook. Pharmacotherapy, 36(5), 511-535. doi: 10.1002/phar.1747.

Skidmore, C. R., Kaufman, E. A., \& Crowell, S. E. (2016). Substance Use Among College Students. Child and Adolescent Psychiatric Clinics of North America, 25(4), 735-53. doi: 10.1016/j. chc.2016.06.004

Sobell, L. C., \& Sobell, M. B. (1992). Timeline follow-back: A technique for assessing self-reported alcohol consumption. En R. Z. Litten,
\& J. P. Allen (Eds.), Measuring alcohol consumption: Psychosocial and biochemical methods (pp. 41-72). Humana Press. doi: 10.1007/978-1-4612-0357-5_3

Szerman, N., Martinez-Raga, J., Peris, L., Roncero, C., Basurte, I., Vega, P., Ruiz, P., \& Casas, M. (2013). Rethinking Dual Disorders/ Pathology. Addictive Disorders \& Their Treatment, 12(1), 1-10. doi: 10.1097/ADT.0b013e31826e7b6a

Tiburcio, M., Rosete-Mohedano, M. G., Natera, G., Martínez, N. A., Carreño, S., \& Pérez, D. (2016). Validity and Reliability of the Alcohol, Smoking, and Substance Involvement Screening Test (ASSIST) in University Students. Adicciones, 28(1), 19-27. doi 10.20882/adicciones.786

United Nations Office on Drugs and Crime. (2017). Global Overview of Drug Demand and Supply: Latest trends, cross-cutting issues. Recuperado de https://www.unodc.org/wdr2017/field/Booklet_2_ HEALTH.pdf

Useche, F. A., Escalona, M. J., López, V. M., \& Ortiz, G. J. (2017). Factores psicosociales en la recaída de la dependencia al alcohol: Un análisis de ruta. Enfermería Investiga, 2(4), 137-144. doi: 10.29033/ei.v2n4.2017.04

Villalobos, L., Pérez, A., Mendoza, R., Graue, J., \& Marín, R. (2015). Psychometric and diagnostic properties of the Drug Abuse Screening Test (DAST): Comparing the DAST-20 vs. the DAST-10. Salud Mental, 38(2), 89-94. doi: 10.17711/SM.01853325.2015.012

Villatoro-Velázquez, J. A., Oliva, N., Fregoso, D., Bustos, M., Mujica, A., Martín del Campo, R., Nanni, R., \& Medina-Mora M. E. (2015). Encuesta Nacional de Consumo de Drogas en Estudiantes: Reporte de Drogas. Secretaría de Salud.

Vujanovic, A. A., Meyer, T. D., Heads, A. M., Stotts, A. L., Villarreal, Y. R., \& Schmitz, J. M. (2017). Cognitive-behavioral therapies for depression and substance use disorders: An overview of traditional, third-wave, and transdiagnostic approaches. The American Journal of Drug and Alcohol Abuse, 43(4), 402-415. doi: 10.1080/00952990.2016.1199697 